\title{
artigo
}

\section{Perfil sociodemográfico de notificação de acidentes de trabalho com material biológico}

\author{
Sociodemographic profile of notification of occupational accidents with biological material \\ Perfil sociodemográfico de notificación de accidentes laborales con material biológico
}

\section{RESUMO}

Objetivo: Descrever o perfil sociodemográfico dos acidentados com material biológico no município de Araruama. Métodos: Trata-se de uma pesquisa documental, de cunho descritivo e abordagem quantitativa. Delimita-se como cenário do estudo o Programa de Saúde do Trabalhador Municipal, setor que recebe todas as notificações de acidente com material biológico, sendo então lançadas no Sistema de Informação de Agravos de Notificação e investigadas. Resultados: Constatou-se que houve no período do corte temporal 319 notificações, em sua maioria (76\%) os acidentes ocorreram com mulheres, sendo a enfermagem o grupo mais acometido, especificamente os técnicos de enfermagem. Por auto declaração determinou-se que a maior parte dos acidentados era da raça branca. Considerações Finais: Um longo caminho ainda deve ser trilhado em relação à segurança nos ambientes de trabalho e atendimento dos acidentados, onde deve-se ressaltar o valor único da vida e resguardar os direitos dos trabalhadores, enfatizando a enfermagem, por sua importância e quantitativo nas unidades de saúde.

DESCRITORES: Enfermagem; Notificação de Acidentes de Trabalho; Exposição Ocupacional; Risco por Agentes Biológicos; Sistemas de Informação em Saúde.

\section{ABSTRACT}

Objective: To describe the sociodemographic profile of those injured with biological material in the municipality of Araruama. Methods: This is a documentary research, of a descriptive nature and quantitative approach. The Municipal Worker's Health Program is delimited as the study scenario, a sector that receives all notifications of accidents with biological material, and is then launched in the Information System for Notifiable Diseases and investigated. Results: It was found that there were 319 notifications in the time period, most of them $(76 \%)$ accidents occurred with women, with nursing being the group most affected, specifically nursing technicians. By self declaration it was determined that most of the injured were white. Conclusion: There is still a long way to go in relation to safety in the workplace and care for the injured, where the unique value of life and the protection of workers' rights must be emphasized, emphasizing nursing, due to its importance and quantity in health units. DESCRIPTORS: Nursing; Notification of Accidents at Work; Occupational Exposure; Risk by Biological Agents; Health Information Systems.

\section{RESUMEN}

Objetivo: Describir el perfil sociodemográfico de los heridos con material biológico en el municipio de Araruama. Métodos: Se trata de una investigación documental, de carácter descriptivo y enfoque cuantitativo. Se delimita como escenario de estudio el Programa de Salud del Trabajador Municipal, sector que recibe todas las notificaciones de accidentes con material biológico, y luego se lanza en el Sistema de Información de Enfermedades Notificables e investiga. Resultados: Se encontró que hubo 319 notificaciones en el período de tiempo, la mayoría de ellos (76\%) accidentes ocurrieron con mujeres, siendo la enfermería el grupo más afectado, específicamente los técnicos de enfermería. Por autodeclaración se determinó que la mayoría de los heridos eran blancos. Conclusión: Aún queda un largo camino por recorrer en materia de seguridad en los ambientes laborales y atención al lesionado, donde se debe enfatizar el valor único de la vida y la protección de los derechos de los trabajadores, destacando la enfermería, por su importancia y cantidad en Unidades de salud.

DESCRIPTORES: Enfermería; Notificación de accidentes laborales; Exposición ocupacional; Riesgo por agentes biológicos; Sistemas de información sanitaria.

RECEBIDO EM: 30/10/2020 APROVADO EM: 10/11/2020 


\section{Patrícia Dias Barreto Marinho}

Possui graduação em Enfermagem pela Universidade Veiga de Almeida (2007). Especialização em Enfermagem do trabalho pela Universidade Federal do Rio de Janeiro ( 2009).Mestre pelo programa MPEA da Universidade Federal Fluminense . Professora da Faculdade União Araruama de Ensino.

ORCID: 0000-0002-8822-9293

\section{Cristina Lavoyer Escudeiro}

Doutora em Enfermagem (2002)- UFRJ, coordenadora da graduação em Enfermagem - UFF na EEAAC, Professora Associada na EEAAC. Concluiu o doutorado em publicou 30 artigos em periodicos especializados e 160 trabalhos em anais de eventos. Possui 3 livros publicados.

ORCID: 0000-0001-8920-5245

\section{INTRODUÇÃO}

0 trabalho mostra-se como forma de autoafirmação do sujeito, inserindo-o em um contexto social, meio de prover seu sustento e obter realização pessoal. O modo como o homem e o trabalho interagem vem sofrendo profundas transformações. A globalização e as novas tecnologias têm contribuído para essa forma multifacetada, apresentada pelos profissionais, na busca constante do aperfeiçoamento e para suprir as necessidades de mercado. ${ }^{(1)}$ Tais tecnologias facilitam a vida do trabalhador, porém, atribuem a este uma sobreposição nas suas atribuições habituais, ao ter que se adequar adaptá-las ao seu processo de trabalho.

O trabalho é aspecto fundamental da vida humana. Do ponto de vista dos indivíduos, o trabalho condiciona um modo de vida; a profissão das pessoas as define e é parte intrínseca de sua personalidade. Já das relações, o trabalho desempenhado situa as pessoas numa hierarquia social de valores e predeterminam toda uma série de expectativas. Mais ainda, o trabalho e sua remuneração são repletos de significados conscientes e inconscientes ${ }^{(2)}$.

No trabalho do profissional da área de saúde, e da enfermagem, os significados estão entrelaçados com a exposição constante a vírus, bactérias e riscos decorrentes do desempenho de suas funções laborais.

No que tange à saúde do trabalhador, esta área da saúde pública teve sua nomenclatura reconhecida no Brasil após a Reforma Sanitária, com as mudanças ocorridas nas décadas de 80 e 90 . A promulgação da Constituição Federal de 1988 e a Lei Orgânica da Saúde consideram que a saúde deixou de ser destinada às classes trabalhistas específicas e passou a ser "direito fundamental". Se consolidando posteriormente como política de saúde e em área específica do Ministério da Saúde ${ }^{(3)}$.

O presente estudo aborda mais especificamente a notificação de acidente de trabalho com exposição a material biológico; levando-se em consideração que a enfermagem se configura como um grupo de trabalho numérico nas instituições hospitalares.

Atualmente, os esforços para manter o trabalhador de enfermagem saudável mostram-se essenciais, baseado na peculiaridade do trabalho e aos riscos que o mesmo é exposto em sua atividade laboral. Os estudos mostram a importância de elaboração e implantação de medidas preventivas para o ambiente de trabalho hospitalar, considerado como um local onde este ator social se expõe a vírus, bactérias e outros micro-organismos no desempenho de suas atividades laborais, configurando assim a possibilidade de exposição biológica que o trabalhador apresenta ${ }^{(4)}$.

Segundo estimativas de outros órgãos internacionais, acontecem 3 milhões de exposições perfurocortantes com material biológico anualmente, entre 35 milhões de profissionais ao redor do mundo ${ }^{(4,5)}$.

O sujeito que exerce a ação laborativa no contexto da saúde necessita ter resguardado o seu direito as condições adequadas e seguras de trabalho, minimizando os riscos de exposição ocupacional a agentes biológicos e prezando pelos deveres das instituições de saúde nesta relação, fato que está disposto na Norma Regulamentadora n. $32^{(5)}$.

A relevância deste estudo está embasada no direito que o trabalhador de enfermagem possui de exercer seu trabalho com medidas salubres de proteção e minimização de riscos, tendo conhecimento das possibilidades e doenças que pode ser acometido em seu processo de trabalho. Diante deste aspecto delimita-se como questão norteadora da pesquisa: Qual o perfil sociodemográfico dos acidentes com material biológico no município de Araruama?

Dado o exposto, objetivou-se descrever o perfil sociodemográfico dos acidentados com material biológico no município de Araruama

\section{MÉTODOS}

Trata-se de um estudo descritivo, de abordagem quantitativa, oriundo de pesquisa documental. A abordagem quantitativa obtém dados descritivos através de um método estatístico. Esta análise é mais objetiva e mais exata, fundamenta-se na frequência de aparição de determinados elementos na mensagem. ${ }^{(6)}$ O cenário do estudo é o Programa de Saúde do Trabalhador Municipal de Araruama, setor que recebe todas as notificaçôes de acidente com material biológico, onde serão, então, lançadas no SINAN e investigadas. Fisicamente, fica situado no Núcleo de Saúde Coletiva, repartição municipal que é responsável por coordenar os programas de saúde pública, onde está localizada a vigilância ambiental, sanitária e epidemiológica. Todos os programas abastecem seus dados nos respectivos sistemas de informação, no caso do Programa de Saúde do Trabalhador, todas as notificações de acidentes de trabalho, doenças relacionadas ao trabalho e intoxicações são lançadas no SINAN, juntamente com alguns outros agravos de notificação compulsória.

No estudo, a amostra comtemplou que fossem analisadas todas as fichas de acidente por material biológico do período de 01 de janeiro de 2010 a 01 setembro de 2015, o que totalizou 333 formulários de notificação de acidentes com material biológico. Foram 
considerados para análise 319 formulários de notificação após aplicação do critério de exclusão. A justificativa para a análise de todas as fichas deste corte temporal deu-se pelo fato de que a enfermagem está constantemente exposta a riscos no desenvolvimento de seu trabalho, acrescentando ainda a exposição do profissional a vários estressores ambientais, de natureza biológica, física, química ou ligada à organização do trabalho.(7)

Os critérios de inclusão para os documentos foram: ser notificação do agravo acidente de trabalho com material biológico, estar dentro do corte temporal, ser de unidades do município de Araruama. Foram utilizadas como critério de exclusão as notificações sem o preenchimento do item Ocupação.

A coleta de dados ocorreu por meio de um levantamento de dados secundários dos acidentes por materiais biológicos, realizado nos arquivos existentes no setor de saúde coletiva, onde ficam conservadas todas as fichas de notificações dos agravos notificáveis. Posteriormente, foi realizada busca no sistema SINAN, com o número de cada notificação existente nos arquivos, para conferir se estavam devidamente lançadas no sistema. No que se refere ao instrumento utilizado para a coleta de dados, trata-se de formulário de notificação compulsória, com o Código Internacional de Doenças número Z20.9, que se encontram em todas as unidades de saúde que estejam amparadas pela NR 32.

Os dados coletados foram analisados por meio da estatística descritiva que permite ao pesquisador resumir e descrever dados quantitativos, sendo as distribuições por frequência ordenada através do valor obtido na coleta de dados.

O presente estudo é um recorte da pesquisa "Desenvolvimento de software para acompanhamento da saúde do trabalhador de enfermagem do município de Araruama”, aprovada pelo Comitê de Ética em Pesquisa da Faculdade de Medicina da Universidade Federal Fluminense (CEP/FM/UFF) sob o parecer n. 911.973 , com número de aprovação 35037914.3.0000.5243.

\section{RESULTADOS}

Foram analisadas 319 fichas de notificação de acidente de trabalho com exposição a ma- terial biológico no período de 2010 a 2015. Durante os anos de 2011, 2012 e 2014 a média de acidentes correspondeu a aproximadamente $22 \%, 22 \%$ e $20 \%$ respectivamente, apresentando os maiores picos de frequência.

Os dados levantados nas notificações e no Sistema Nacional de Agravos Notificáveis, relativas ao município de Araruama, expõem o quantitativo de acidentes que foram notificados, não sendo possível, no entanto, precisar a quantidade de subnotificação. Observa-se que o mês de novembro de 2011 apresenta um pico de 18 notificações, seguido por outubro de 2014 com 13 casos. Não houve uma constância em relação aos mesmos meses em anos diferentes, que caracteriza um pico das notificaçôes. Houve meses isolados com aumento significativo e decréscimo em outros períodos do ano. Tal quantitativo certamente seria de maior significância, não fossem as subnotificações.

Em relação à distribuição de sexo nos casos de notificação, mostra-se, como apresentado na Tabela 1 , o alto índice de acidentes na população feminina. $\mathrm{O}$ que pode evidenciar que a maior força de trabalho na saúde, nesta localidade, é de mulheres, ou ainda, as mulheres preocupam-se mais com a realização de exames e notificações, quando são acometidas por um acidente durante seu processo de trabalho.

A média de mulheres acometidas nos eventos de acidente por material biológico em que houve notificação varia entre $62 \%$ e $80 \%$, com uma média de todos os anos de $74 \%$ de atores sociais do sexo feminino que sofreram acidentes biológicos.
Em relação à distribuição da frequência por idade do profissional acidentado, verificou-se uma predominância na faixa etária de 40 a 50 anos (35\%), seguida da faixa compreendida entre 18 e 38 anos, com uma frequência de $28 \%$. A idade compreendida entre 29 e 39 anos foi responsável por $25 \%$ das notificações, seguida por pessoas acima de 51 anos com $12 \%$.

Segundo a ocupação, observa-se grande significância e relevância o quantitativo de técnicos de enfermagem que sofreu acidente por material biológico, despontando como a ocupação que mais sofreu tal injúria ocupacional com um $n=175$ (54,86\%). Os enfermeiros apresentaram 19 casos e um notificação de enfermeiro do trabalho. A enfermagem como a maior força de trabalho dos hospitais e instituiçôes de saúde, que tem como essência de sua profissão prestar o cuidado direto ao cliente, algumas vezes torna-se mais suscetível a alguns tipos de exposições. Os auxiliares de enfermagem apresentaram um $n=03$, médicos clínicos e suas notificações expressam um $\mathrm{n}=20$, com $6,3 \%$ de representatividade, frente aos médicos cirurgiões e legistas, com um n de uma notificação cada um.

\section{DISCUSSÃO}

O acidente de trabalho com material biológico caracteriza-se como o contato de vírus, bactérias e patógenos através de sangue e secreções contaminadas através da atividade laborativa, ressalta-se ainda que estão vinculados à transmissão de patógenos virais como Hepatite B, C e HIV. ${ }^{(8)}$

Em relação à distribuição aos acidentes

\section{Tabela 1. Distribuição da frequência de notificações segundo o sexo.}

\begin{tabular}{ccccccc}
\hline \multirow{2}{*}{ Ano da Notificação } & \multicolumn{2}{c}{ Masculino } & \multicolumn{2}{c}{ Feminino } & Total & $\%$ \\
\hline 2010 & N & $\%$ & N & $\%$ & & \\
2011 & 8 & 20 & 33 & 80 & 41 & 100 \\
2012 & 15 & 21 & 55 & 79 & 70 & 100 \\
2013 & 19 & 27 & 52 & 73 & 71 & 100 \\
2014 & 12 & 23 & 40 & 77 & 52 & 100 \\
2015 & 21 & 33 & 43 & 67 & 64 & 100 \\
Total & 8 & 38 & 13 & 62 & 21 & 100 \\
Fonte: elaborado pela autora. & 83 & 26 & 236 & 74 & 319 & 100 \\
\hline
\end{tabular}




\section{Tabela 2. Distribuição da frequência por situação no mercado de trabalho segundo a ocupação.}

INVESTIGAÇÃO DE ACIDENTE COM MATERIAL BIOLÓGICO

\section{Frequência por Situação no Mercado de Trabalho segundo a Ocupação}

\section{Ocupação}

Mecânico de manutenção de automóveis

Auxiliar geral de conservação de vias

Estudante

Dona de casa

Soldado da polícia militar/guarda civil

Biólogo

Médico cirurgião geral

Médico clínico

Médico legista

Cirurgião dentista-clínico geral

Enfermeiro

Enfermeiro do trabalho

Fisioterapeuta

Psicólogo hospitalar

Técnico de enfermagem

Auxiliar de enfermagem

Técnico em higiene dental/aux. Prótese dentária

Técnico em radiologia e imagenologia

Técnico em laboratório de farmácia

Auxiliar de pessoal

Empregado doméstico nos serviços gerais

Coletor de lixo

Faxineiro

Gari

Auxiliar de laboratório de análises clínicas

Atendente de farmácia-balconista

Abatedor

Total

Fonte: elaborado pela autora

Legenda: A- Ignorado/ Branco; B- Empregado registrado; C- Empregado não registrado; D- Autônomo; E- Servidor público estatutário; F- Servido público celetista; G- Desempregado; H- Trabalhador temporário; I- Cooperativado; J- Trabalhador Avulso; K- Outros. por sexo, corrobora os resultados encontrados, o fato de o maior número de acidentes acometer trabalhadores do sexo feminino. ${ }^{(9-11)}$ Ressalta-se ainda que, como nos demais estudos que alicerçam a pesquisa, o número de acidentados do sexo feminino ultrapassou os $70 \%$, onde pode ter explicação na história formativa de cada profissão. ${ }^{(12)}$

No resultado referente à idade, há uma

$\begin{array}{ccccccccccccc}\text { A } & \text { B } & \text { C } & \text { D } & \text { E } & \text { F } & \text { G } & \text { H } & \text { I } & \text { J } & \text { K } & \text { Total } & \% \\ 1 & 0 & 0 & 0 & 0 & 0 & 0 & 0 & 0 & 0 & 0 & 1 & 0,31 \\ 0 & 1 & 0 & 0 & 0 & 0 & 0 & 0 & 0 & 0 & 0 & 1 & 0,31 \\ 4 & 1 & 0 & 1 & 0 & 0 & 0 & 2 & 0 & 0 & 8 & 16 & 5,01 \\ 1 & 0 & 0 & 0 & 0 & 0 & 0 & 0 & 0 & 0 & 0 & 1 & 0,31 \\ 0 & 0 & 0 & 0 & 1 & 0 & 0 & 0 & 1 & 0 & 0 & 2 & 0,62 \\ 0 & 0 & 1 & 0 & 0 & 0 & 0 & 0 & 0 & 0 & 0 & 1 & 0,31 \\ 0 & 1 & 0 & 0 & 0 & 0 & 0 & 0 & 0 & 0 & 0 & 1 & 0,31 \\ 3 & 5 & 0 & 0 & 0 & 2 & 0 & 3 & 0 & 0 & 7 & 20 & 6,3 \\ 0 & 0 & 0 & 0 & 1 & 0 & 0 & 0 & 0 & 0 & 0 & 1 & 0,31 \\ 1 & 0 & 1 & 1 & 0 & 0 & 0 & 2 & 0 & 1 & 0 & 6 & 1,88 \\ 5 & 3 & 1 & 0 & 3 & 0 & 0 & 6 & 0 & 0 & 1 & 19 & 5,96 \\ 0 & 0 & 0 & 0 & 0 & 1 & 0 & 0 & 0 & 0 & 0 & 1 & 0,31 \\ 1 & 1 & 0 & 0 & 0 & 1 & 0 & 4 & 0 & 0 & 0 & 7 & 2,2 \\ 0 & 0 & 0 & 0 & 0 & 0 & 0 & 2 & 0 & 0 & 0 & 2 & 0,62 \\ 18 & 28 & 4 & 1 & 12 & 17 & 1 & 46 & 35 & 1 & 12 & 175 & 54,86 \\ 0 & 0 & 0 & 0 & 2 & 1 & 0 & 0 & 0 & 0 & 0 & 3 & 0,94 \\ 0 & 3 & 0 & 0 & 0 & 0 & 0 & 1 & 0 & 0 & 1 & 5 & 1,56 \\ 0 & 1 & 0 & 0 & 0 & 0 & 0 & 0 & 0 & 0 & 0 & 1 & 0,31 \\ 1 & 0 & 0 & 0 & 0 & 0 & 0 & 0 & 0 & 0 & 0 & 1 & 0,31 \\ 0 & 2 & 0 & 0 & 0 & 1 & 0 & 2 & 1 & 0 & 0 & 6 & 1,88 \\ 0 & 1 & 0 & 1 & 0 & 0 & 0 & 0 & 0 & 0 & 0 & 2 & 0,62 \\ 1 & 22 & 2 & 0 & 0 & 0 & 0 & 0 & 1 & 0 & 1 & 27 & 8,48 \\ 1 & 6 & 0 & 0 & 0 & 1 & 0 & 2 & 0 & 0 & 2 & 12 & 3,79 \\ 0 & 2 & 0 & 0 & 0 & 0 & 0 & 0 & 0 & 0 & 0 & 2 & 0,62 \\ 1 & 1 & 0 & 0 & 0 & 0 & 0 & 0 & 1 & 0 & 0 & 3 & 0,94 \\ 0 & 1 & 0 & 0 & 0 & 0 & 0 & 0 & 0 & 0 & 0 & 1 & 0,31 \\ 2 & 0 & 0 & 0 & 0 & 0 & 0 & 0 & 0 & 0 & 0 & 2 & 0,62 \\ 40 & 79 & 10 & 4 & 19 & 24 & 1 & 71 & 38 & 1 & 32 & 319 & 100\end{array}$

0,62

1,56

,31

1,88

8,62

, 79

0,62

94

0,62

100 prevalência de acidentados de 40-50 anos. Este número diverge das pesquisas que apresentaram prevalência na faixa etária de 30-40 anos, ${ }^{(9)}$ e 20-30 anos. ${ }^{(13-15)}$

A escolaridade apresentou um índice de $47 \%$ de fichas onde não foi preenchido esse item, o que dispara um alerta significativo para a qualidade do preenchimento das fichas de notificação. Conforme levantamento, o grupo que mais apresentou acidentes biológicos foram os de nível médio. ${ }^{(1,13,16)}$

As ocupações e situação no mercado de trabalho expostos mostram um grande quantitativo de técnicos de enfermagem acidentados e, sequencialmente os coletores de lixo. A incidência de acidentes biológicos com os técnicos de enfermagem chegou aos 26\% em 2010. Segundo pesqui- 


\section{artigo}

sas, ${ }^{(16)}$ questões como o descarte do perfurocortante e a sobrecarga de trabalho são motivos que justificam estes números. ${ }^{(10)}$

O trabalho da enfermagem e dos profissionais de saúde exige uma boa comunicação, pois neste espaço interagem recursos humanos atuando com recursos materiais, em um espaço físico nem sempre apropriado, os quais devem ser gerenciados de maneira adequada visando ao bem-estar de pacientes e trabalhadores. ${ }^{(17,18)}$

Alguns fatores e situações de trabalho, no ambiente hospitalar, predispõem ou acentuam as possibilidades de acidentes e doenças ao trabalhador pela exposição ao risco biológico.
Nas unidades de emergência, por exemplo, onde existe maior exposição aos riscos, a exposição ocupacional pode aumentar em até $85 \%$. Além disso, o número insuficiente de trabalhadores, a sobrecarga e jornadas fatigantes de trabalho, a continuidade da assistência expressa por turnos e plantões noturnos, o desgaste físico e emocional, a falta de capacitação profissional e a cultura local são fatores muito importantes a se considerar. ${ }^{(19)}$

\section{CONCLUSÃO}

Buscou-se conhecer o perfil dos trabalhadores acidentados, a fim de ter um panorama fidedigno para implementação de estratégias para melhoria da qualidade de vida do trabalhador e resguardar o indivíduo em sua integralidade, evitando ou minimizando os danos de um possível processo saúde-doença decorrente das atividades laborais.

Como esperado, a equipe de enfermagem foi a mais acometida pelos acidentes com materiais biológicos e, em sua maioria, mulheres, trazendo à tona o histórico da profissão. Enfatiza-se a existência de sobrecarga de trabalho para a enfermagem, sendo a falta de tempo para a realização da notificação uma das causas para a subnotificação de casos, consistindo em uma limitação deste estudo.

\section{REFERÊNCIAS}

1. Ribeiro RP, Martins JT, Marziale MHP, Robazzi MLCC. O adoecimento pelo trabalhado de enfermagem: uma revisão integrativa. Rev latino-am enferm. 2012; 46(2):495-504.

2. Garanhani ML, Martins JT, Robazzi MLCC, et al. O trabalho de enfermagem em unidade de terapia intensiva: significados para técnicos de enfermagem. SMAD Rev eletrônica saúde mental alcool drog. 2008 ago;4(2):01-15.

3. Jacques CC, Milanez B, Mattos RCO. Indicadores para centro de referência de saúde do trabalhador: proposição de um sistema de acompanhamento dos serviços de saúde. Ciênc saúde coletiva. $2012 ; 17(2): 369-78$.

4. Schimidt DRC, Dantas RAS, Marziale MHP. Ansiedade e depressão entre profissionais de enfermagem que atuam em blocos cirúrgicos. Rev Esc Enferm USP. 2011;45(2):487-93.

5. Gaze R, Leão LHC; Vasconcellos LCF. A Organização Internacional do Trabalho: a saúde fora do lugar. In: Vasconcellos LCF.; Barros M.H. Saúde, trabalho e direito: uma trajetória crítica e a crítica de uma trajetória. Rio de Janeiro: Educam, 2011; 201-256.

6. Figueiredo, TO. Construção do software "sistema de indicadores de gestão do capital humano de enfermagem em cenário hospitalar" 2014. 125 f. Dissertação (Mestrado Profissional em Enfermagem Assistencial) - estudo metodológico. Escola de Enfermagem Aurora de Afonso Costa, Universidade Federal Fluminense, Niterói, 2014.

7. Lima DVM. Research design: a contribution to the author. Online braz j nurs. 2011 Oct; 10(2).

8. Medeiros EAS, Bakolswi E, Sassi SJG, Destra AS. Eventos adversos relacionados à profilaxia anti-retroviral em acidentes ocupacionais. Rev saúde pública. 2007;41(2):294-92

9. Simão, SAF. Perfil dos acidentes ocupacionais com material biológico entre profissionais de saúde. 2010. 88 f. Dissertação (Mestrado em Ciências do Cuidado em Saúde) - Escola de Enfermagem Aurora de Afonso Costa, Niterói, 2010.

10. Paulino DCR, Lopes MVO, Rolim ILTP. Biossegurança e aci- dentes de trabalho com perfuros cortantes entre profissionais de enfermagem de Hospital universitário de Fortaleza. Cogitare enferm. 2008 out-dez; 13(4):507-13.

11. Ferreira MD, Pimenta FR, Facchin LT, Gir E,Canini SRMS. Subnotificação de acidentes biológicos pela enfermagem de um hospital Univesitário. Cienc enferm. 2015;2:21-9

12. Spagnuolo RS, Baldo RCS, Guerrini IA. Análise epidemiológica dos acidentes com material biológico registrados no Centro de Referência em Saúde do Trabalhador - Londrina-PR. Rev bras epidemiol. 2008;11(2):315-23.

13. Valim MD, Marziale MHP. Avaliação da exposição ocupacional a material biológico em serviços de saúde. Texto \& contexto enferm. 2011;20(spe):138-46.

14. Silva AICD. Análise da qualidade dos registros de acidentes biológicos com matérias perfurocortantes na Fundação Osvaldo Cruz no período de 1999 a 2004. 2006. 80 f. Dissertação (Mestrado Profissional em Vigilância em Saúde) - Escola Nacional de Saúde Pública, Fundação Osvaldo Cruz, Rio de Janeiro, 2006.

15. Valim MD, Marziale MHP, Hayashida M, Martinez MR. Ocorrência de acidentes de trabalho com material biológico potencialmente contaminado em enfermeiros. Acta Paulista de Enfermagem. 2014;27(3):280-6.

16. Oliveira AC, Paiva MHR. Condutas pós acidente ocupacional por exposição a material biológico entre profissionais de serviço de urgência. Rev enferm UERJ. 2014 jan-fev;22(1):116-22.

17. Galdino A, Santana VS, Ferrite S. Os centros de referência e o acidente biológico. Cad. Saude pública. 2012 jan;28(1):145-159.

18. Pimenta FR, Ferreira MD, Gir E, Hayashida M. Atendimento e seguimento clínico especializado de profissionais de enfermagem acidentados com material biológico. Rev Esc Enferm USP. 2013;47(1):198-204.

19. Silva MKD, Zeitoune RCG. iscos ocupacionais em um setor de hemodiálise na perspectiva dos trabalhadores da equipe de enfermagem. Esc Enferm Anna Nery. 2009;13(2):279-286. 